\title{
Lesión de Dieulafoy cecal: una causa inusual de rectorragia severa recurrente
}

\author{
Jorge Sandoval-Montero ${ }^{1 *}$, Shirley Barboza-Porras ${ }^{2}$ y Jorge Vargas-Madrigal ${ }^{2}$ \\ ${ }^{1}$ Posgrado de Gastroenterología y Endoscopia Digestiva, Universidad de Costa Rica; Gastroenterología y Endoscopia Digestiva, Servicio de \\ Gastroenterología, Hospital Dr. Enrique Baltodano Briceño. Guanacaste, Costa Rica
}

\section{Resumen}

La lesión de Dieulafoy consiste en la persistencia del calibre de una arteria submucosa que protruye sobre una mucosa normal, siendo inusual su ubicación en colon, que ocurre en el $2 \%$ de los casos. Presentamos el caso de un paciente de 30 años, conocido sano, referido por rectorragia severa, con esofagogastroduodenoscopia inicial sin causa de sangrado activo y una colonoscopia que documenta pólipo pediculado de aproximadamente $20 \mathrm{~mm}$ de diámetro, cubierto con restos hemáticos, el cual no parece presentar sangrado activo. Se reprograma 48 horas después para polipectomía y se reseca la lesión, sin evidenciar otras causas de sangrado y verificando adecuada hemostasia. El paciente reconsulta 6 meses después por rectorragia severa y se realiza nueva colonoscopia con hallazgo en el ciego de lesión vascular compatible con lesión de Dieulafoy, la cual se coagula con plasma argón tras un intento fallido de hemostasia con pinza de coagulación. El tratamiento de elección es endoscópico. En caso de fracaso se deberá recurrir a la cirugía y la arteriografía es una buena alternativa en pacientes no candidatos a cirugía.

Palabras clave: Lesión de Dieulafoy. Rectorragia severa. Coagulación con plasma argón (APC).

\section{Lesion of cecal Dieulafoy: an unusual cause of recurrent severe rectal bleeding}

\begin{abstract}
Dieulafoy lesion consists in the persistence of the caliber of a submucosal artery until it protrudes on a normal mucosal; its location in the colon is very unusual and occurs in only $2 \%$ of the cases. We present the case of a 30-year-old man, with no other illness, referred because of severe rectal bleeding, with a normal initial esophagogastroduodenoscopy, and an initial colonoscopy where a $20 \mathrm{~mm}$ pedunculated polyp without recent bleeding signs was detected, covered with blood stains. Due to this finding colonoscopy was repeated in 48 hours and polyp was resected, with adequate hemostasis. Patient reconsulted for severe rectal bleeding, for this reason a new colonoscopy was performed, finding a vascular lesion in the cecum compatible with a Dieulafoy lesion, which was coagulated with argon plasma after an initial failure of hemostasis with coagulation forceps. The main treatment is endoscopic, with surgery being an option in patients who can't be controlled by endoscopy, although in patients who are not surgical candidates, angiography could be considered an option.
\end{abstract}

Key words: Dieulafoy lesion. Severe rectal bleeding. Argon plasma coagulation (APC).

\section{Correspondencia:}

*Jorge Sandoval-Montero

$300 \mathrm{~m}$. Oeste de la Iglesia católica en San

Rafael de Alajuela

Fecha de recepción: 28-01-2019

Fecha de aceptación: 17-02-2019

E-mail: jor_san88@ hotmail.com

DOI: 10.24875/END. 19000003
Disponible en internet: 06-05-2019 Endoscopia. 2019;31:85-89 www.endoscopia-ameg.com 0188-9893/๑ 2019. Asociación Mexicana de Endoscopia Gastrointestinal, publicado por Permanyer México SA de CV, todos los derechos reservados. 


\section{Introducción}

La lesión de Dieulafoy consiste en la persistencia del calibre de una arteria submucosa que protruye sobre una mucosa normal. Es una causa infrecuente de hemorragia digestiva, que se reporta en el 1 al $2 \%$ todos de los casos, de los cuales el $71 \%$ se localizan en el estómago proximal, a nivel de la curvatura menor, a menos de $6 \mathrm{~cm}$ de la unión esofagogástrica, donde las arterias son de mayor tamaño $0^{1-4}$.

Se han descrito en esófago, duodeno, yeyuno, colon, recto y ano; el colon es de los sitios más infrecuentes, con un $2 \%$, y existen 17 casos reportados hasta el año 2001. Es más frecuente en hombres a una edad media de 50-70 años $2,5,6$.

En 1897 el patólogo Paul Dieulafoy fue el primero en describir dicha condición en una serie de casos de 10 pacientes que presentaron hematemesis masiva secundaria a sangrado de un vaso visible, sin evidencia de ulceración de la mucosa en 3 de esos casos. Mediante autopsia detectó una ulceración superficial que correspondía a la presencia de un vaso submucoso de grueso calibre que protruía hacia la mucosa gástrica, concluyó que correspondía a una entidad distinta de la úlcera gástrica y la describió como exulceratio simplex, la cual posteriormente se llamó lesión de Dieulafoy 1,7,8.

\section{Caso clínico}

Se presenta el caso de un paciente de 30 años, sano, referido por rectorragia severa asociada a inestabilidad hemodinámica. Posterior a la reanimación se realiza esofagogastroduodenoscopia (EGD) el 16/03/2018 que documenta esofagitis erosiva grado B de los Ángeles, con un pólipo diminuto duodenal, el cual se reseca con pinza. Se decide realizar entonces una colonoscopia, en la cual se documenta la presencia de gran cantidad de sangre que tapiza la pared colónica, que no se logra lavar o aspirar, limitando la óptima valoración de la mucosa, sin evidencia de sitios de sangrado activo. A nivel de colon transverso en ángulo hepático presenta un pólipo pediculado de aproximadamente $20 \mathrm{~mm}$ de diámetro, cubierto con restos hemáticos (Fig. 1), el cual no parece tener sangrado activo, aunque la valoración es limitada por los restos hemáticos adheridos a la pared. Se decide reprogramar para mejor evaluación y polipectomía de la lesión tras adecuada preparación intestinal.

El 19/03/2018 se realiza nueva colonoscopia con preparación BOSTON 7/9 donde se realiza polipectomía con colocación de Endoloop ${ }^{\circledR}$. Resto del estudio sin lesiones evidentes.

El paciente reconsulta 6 meses después por nuevo cuadro de rectorragia severa y se realiza nueva EGD sin datos de sangrado. Se programa nueva colonoscopia, que evidencia a nivel de ciego y válvula ileocecal vaso visible con leve hiperemia compatible con lesión de Dieulafoy cecal. Se intenta aplicar terapia térmica con pinza sin éxito inicial, con sangrado arterial posterior a primer intento, por lo cual se procede a aplicar terapia térmica con coagulación con plasma argón (APC) verificando adecuada hemostasia, sin complicaciones inmediatas (Fig. 2).

El paciente es egresado una semana posterior al procedimiento hemodinámicamente estable, sin datos de resangrado.

\section{Patología y fisiopatología}

En condiciones normales, los vasos van disminuyendo su calibre a medida que se aproximan a la mucosa; sin embargo, la lesión de Dieulafoy tiene el mismo calibre hasta alcanzar la submucosa de 2 a $5 \mathrm{~mm}$, dejando el vaso expuesto, con alto riesgo de sangrado ${ }^{2,9}$.

La patogénesis y mecanismos subyacentes a la tortuosidad y persistencia del calibre de las arterias submucosas aún no están bien establecidos, a pesar de múltiples intentos de correlacionar con diversos mecanismos que puedan culminar en la lesión de la mucosa'.

La fibrosis subintimal, la pérdida de las fibras elásticas adyacentes a la pared arterial necrótica, y el adelgazamiento o pérdida de las fibras musculares de la arteria son eventos observados en el punto de la rotura, lo que apoya la teoría de que el proceso patológico final es uno de lento debilitamiento de la pared del vaso con quizá una eventual dilatación localizada, lo cual estaría relacionado a una ectasia vascular, atrofia mucosa y posible injuria isquémica relacionadas con la edad y enfermedades cardiovasculares, las cuales debilitarían un punto inherentemente vulnerable ${ }^{1,10}$.

Entre los diversos mecanismos propuestos se encontraba la sospecha de una dilatación aneurismática, sin embargo, no se ha logrado demostrar, tampoco la presencia de arterioesclerosis ni vasculitis, entre otras, ha demostrado ser causa de la lesión inicial ${ }^{6,7,11}$.

Actualmente los mecanismos más aceptados son: que la pulsación constante dentro de una arteria submucosa grande puede generar presión suficiente hacia el epitelio, lo que resulta en una erosión y rotura del vaso hacia el lumen; otra hipótesis descrita consiste en la necrosis de las paredes del vaso secundario a 


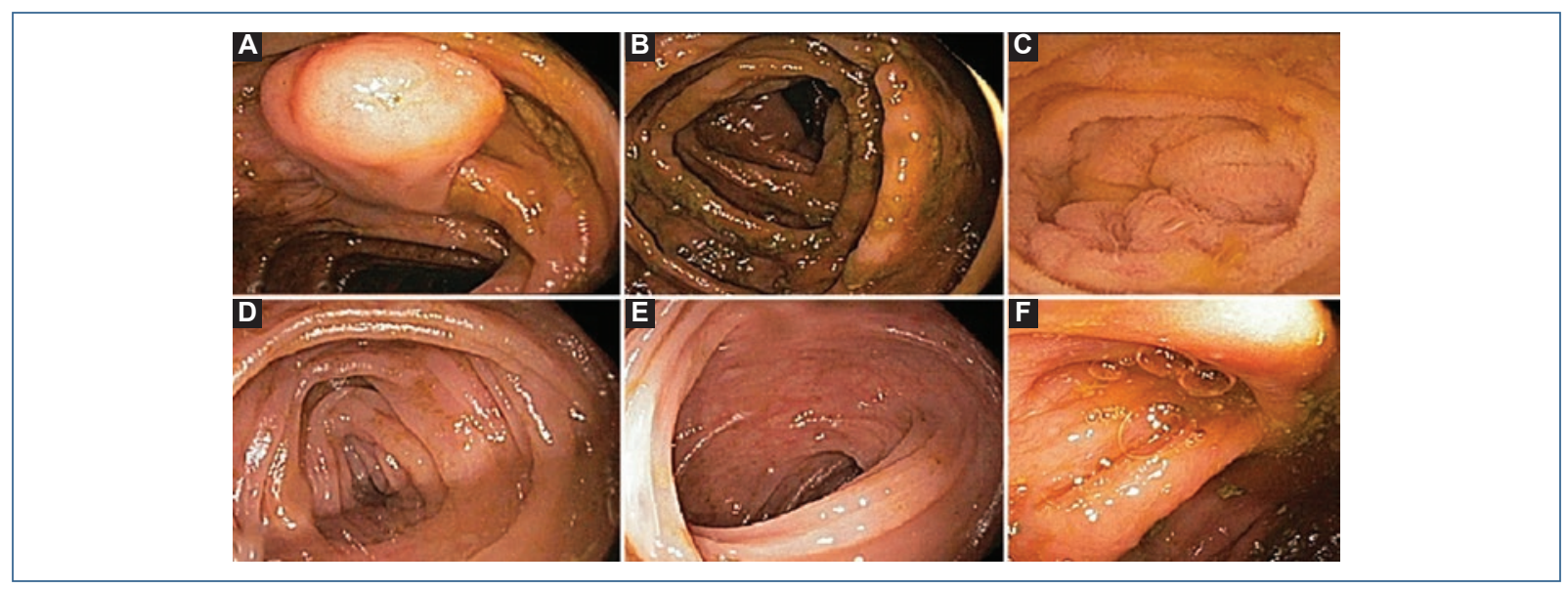

Figura 1. A: pólipo pediculado, posteriormente resecado con Endoloop ${ }^{\circledR}$. B: colon transverso. C: íleon terminal. D: colon descendente. E: colon ascendente. F: apéndice cecal.

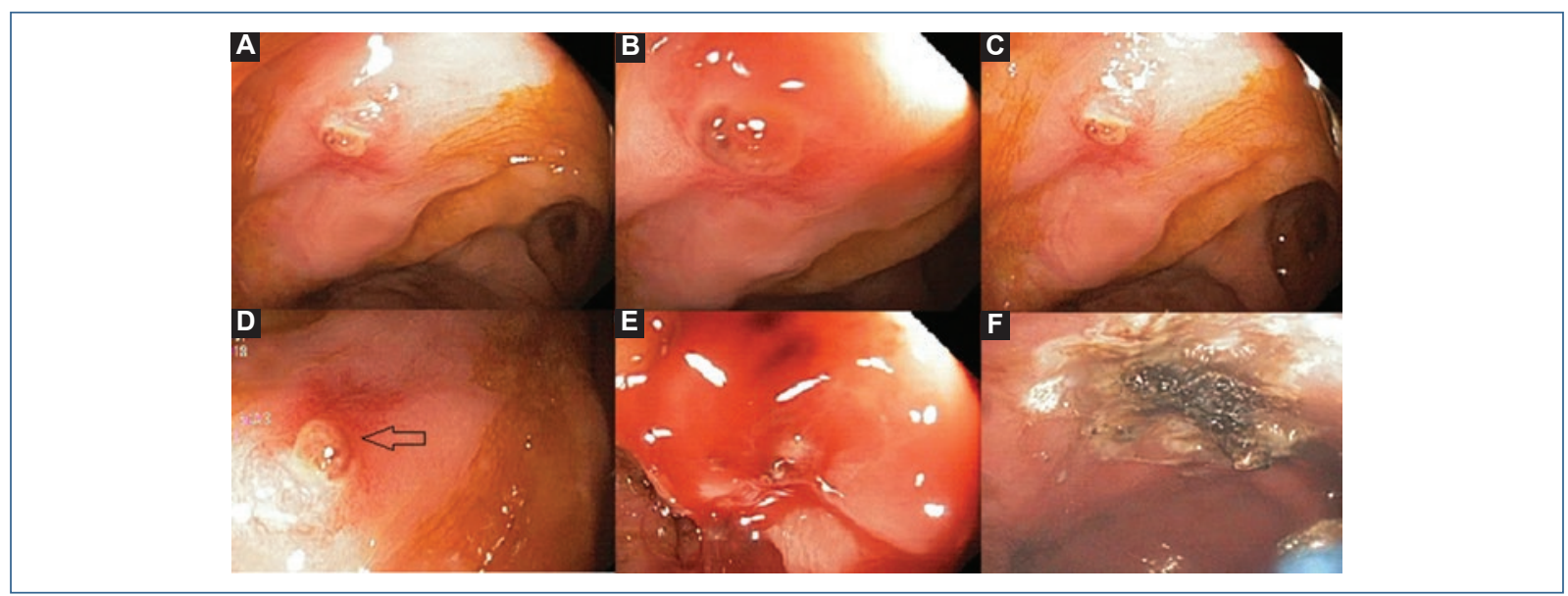

Figura 2. Colonoscopia (A, B, C y D). Lesión de Dieulafoy en ciego, con leve hiperemia perilesional. E: lesión con sangrado activo posterior a intento de hemostasia. F: hemostasia mediante terapia térmica con coagulación con plasma argón.

trombosis dentro de la misma arteria, que suele asociarse a soluciones de continuidad en la mucosa, y consecuente sangrado $0^{9,12}$.

Se desconocen hasta la fecha mutaciones genéticas asociadas a la lesión de Dieulafoy, lo cual sugiere que es un fenómeno adquirido, sin embargo, la localización tan frecuente en cuerpo gástrico puede sugerir un defecto congénito en dicha región, o cambios anatómicos que causen predisposición a desarrollar la lesión $2,10,13$.

\section{Manifestaciones clínicas}

La presentación clínica suele corresponder habitualmente a una hemorragia masiva única o recurrente, usualmente indolora, aunque en raras ocasiones la hemorragia puede estar precedida por síntomas del tracto gastrointestinal y seguida de una hemorragia intermitente durante varios días ${ }^{1-4}$. En el caso de esta revisión el paciente acudió por rectorragia con inestabilidad hemodinámica sin una causa aparente en la EGD inicial y un hallazgo incidental de un pólipo de $20 \mathrm{~mm}$, el cual se resecó, sin otras causas aparentes de sangrado. Tras un periodo de varios meses el paciente reconsulta por rectorragia, con nueva EGD sin causa aparente de sangrado, hasta encontrar en una tercera colonoscopia el hallazgo culpable de la rectorragia en un sitio poco habitual como lo es el ciego. 


\section{Diagnóstico}

El diagnóstico de seguridad solo puede realizarse con el estudio histológico de la pieza quirúrgica. Las características histopatológicas de las lesiones colónicas y de otras localizaciones son similares a las de las lesiones gástricas ${ }^{2}$. La endoscopia puede brindar un alto índice de seguridad; se han definido tres criterios diagnósticos compatibles con lesión de Dieulafoy por vía endoscópica:

- Salida a chorro o flujo micropulsátil de sangre arterial, en forma activa a partir de un defecto mucoso diminuto $(<3 \mathrm{~mm})$ o a través de una mucosa aparentemente normal ${ }^{1,2}$.

- Visualización de un vaso con o sin signos de sangrado reciente, que protruye a través de un defecto mucoso diminuto o a través de mucosa normal ${ }^{1,2}$.

- Presencia de un coágulo fresco fuertemente adherido a través de un estrecho punto de inserción sobre un defecto mucoso diminuto o sobre mucosa de aspecto normal ${ }^{1,2}$.

En nuestro caso, el paciente presentaba un vaso visible con signos de sangrado reciente y posteriormente sangrado micropulsátil debido a la manipulación inicial con pinza de coagulación.

En ocasiones el diagnóstico endoscópico puede ser difícil debido a la mala preparación, con abundantes restos sanguíneos, lesiones de pequeño tamaño, sangrado intermitente y existencia de otra posible causa de sangrado; factores que influyeron en los estudios endoscópicos iniciales. La sensibilidad de la endoscopia inicial para establecer el diagnóstico es variable según los estudios, situándose entre el 49 y el $63 \% 1$.

Cuando el sangrado es importante y no se puede identificar su origen con la endoscopia, la arteriografía puede tener un importante papel diagnóstico y terapéutico ${ }^{8,9}$.

\section{Tratamiento}

El $90 \%$ de los casos de Dieulafoy pueden tratarse mediante endoscopia y el manejo es similar en casos gástricos y extragástricos ${ }^{9}$.

Se han descrito una gran variedad de tratamientos endoscópicos, como la inyección de esclerosantes y/o adrenalina, taponamiento con adhesivo de cianoacrilato, clips metálicos, electrocoagulación con pinza caliente, sonda monopolar o bipolar'.

Aunque la adrenalina se ha utilizado como único método hemostático, debería asociarse a un método hemostático definitivo, térmico, mecánico o quirúrgico para evitar la recidiva, ya que la exclusiva administración de adrenalina no produce la destrucción del vaso ${ }^{7,14}$.

Existe evidencia del uso de terapia térmica con APC como monoterapia en 20 pacientes con Dieulafoy gástricos, utilizando sondas de 2 a $3 \mathrm{~mm}$, de 40 a $60 \mathrm{~W}$ en configuración de la unidad electroquirúrgica, sin evidencia de resangrado, ni otras complicaciones hasta 29 meses después del tratamiento ${ }^{15}$.

La magnitud del sangrado puede llegar a impedir una correcta visualización del colon, en los casos en que fracase el tratamiento endoscópico, y sobre todo si el paciente es mal candidato quirúrgico, podría recurrirse a la embolización angiográfica, para la que se han descrito buenos resultados, aunque la embolización de colon presenta más riesgos de perforación por necrosis isquémica secundaria, debido a la disposición de la vascularización regional, con un elevado componente de vasos terminales y escasez de circulación colateral, a diferencia de lo que sucede en estómago, intestino delgado y recto. Entre el 2 y el $6 \%$ de los pacientes requieren tratamiento quirúrgico $0^{8,9,12}$.

\section{Discusión}

La lesión de Dieulafoy consiste en la persistencia del calibre de una arteria submucosa que protruye sobre una mucosa normal, siendo una causa infrecuente de hemorragia digestiva, la cual se reporta hasta en el 1 a $2 \%$ todos de los casos. Suele ser más frecuente en hombres mayores de 50 años, mucho más frecuente en estómago proximal, hacia curvatura menor, principalmente a $6 \mathrm{~cm}$ de la unión esofagogástrica. Esto sugiere que la lesión inicial podría tener un componente anatómico importante, como la irrigación por vasos de gran calibre o un defecto congénito en dicha zona; también se describe su localización en duodeno, esófago y ano, mientras que la descripción en el colon es sumamente infrecuente, alcanzando apenas un $2 \%$ de los casos de Dieulafoy ${ }^{1,4,9}$.

Se han desarrollado criterios endoscópicos que permiten llegar a un diagnóstico con un alto índice de seguridad, siendo innecesario el uso de histología para confirmar el diagnóstico $0^{1,4,10}$.

El diagnóstico de la lesión puede ser laborioso debido a múltiples factores. En el caso actual se presenta limitación importante de la exploración secundaria a mala preparación intestinal, además del hallazgo distractor de un pólipo avanzado, el cual se resecó sin complicaciones; a pesar de ello el paciente recurrió con rectorragia severa, por lo cual se repitieron estudios endoscópicos, en los cuales se logró documentar 
la presencia de una lesión vascular visible con mucosa circundante normal, sugestiva de Dieulafoy, a la cual se intentó aplicar hemostasia con pinza de coagulación, sin éxito inicial, con posterior sangrado inmediato, por lo cual se procedió a la aplicación de APC sin complicaciones inmediatas $7,9,10,12$.

El paciente evoluciona sin mayores complicaciones y es dado de alta sin necesidad de intervención quirúrgica.

\section{Conclusiones}

La lesión de Dieulafoy es una entidad que potencialmente pone en peligro la vida y debe ser sospechada en pacientes con inicio súbito de sangrado digestivo o episodios recurrentes de este. Existen métodos útiles para su diagnóstico y terapia multimodal endoscópica, quirúrgica 0 angiográfica.

El presente es un caso complejo por la localización tan infrecuente de la lesión de Dieulafoy, que junto a hallazgos iniciales que confundieron el abordaje como lo es el pólipo resecado y la mala preparación inicial reflejan la gran dificultad de detectar una entidad potencialmente letal en un paciente previamente conocido sano. Una vez detectada la lesión se logra brindar un tratamiento óptimo con una adecuada evolución clínica.

\section{Declaración de conflictos de interés}

No se reportan conflictos de intereses.

\section{Fuentes de financiamiento}

Los autores no recibieron patrocinio para la realización del artículo.

\section{Bibliografía}

1. Nguyen DC, Jackson CS. The Dieulafoy's lesion. J Clin Gastroenterol. 2015;49(7):541-9.

2. Baxter M, Aly EH. Dieulafoy's lesion: Current trends in diagnosis and management. Ann R Coll Surg Engl. 2010;92(7):548-54.

3. Kanth R, Mali P, Roy PK. Outcomes in Dieulafoy's lesion: A 10-year clinical review. Dig Dis Sci. 2015;60(7):2097-103.

4. Nojkov B, Cappell MS. Gastrointestinal bleeding from Dieulafoy's lesion: Clinical presentation, endoscopic findings, and endoscopic therapy. World J Gastrointest Endosc. 2015;7(4):295.

5. Mahler MA, Villa G, Roig G. Hemorragia digestiva baja masiva por lesión de Dieulafoy de colon. Reporte de caso con video. Acta Gastroenterol Latinoam. 2017;47(3):216-8.

6. Ma C, Hundal R, Cheng EJ. Colonic Dieulafoy's lesion: A rare cause of lower gastrointestinal hemorrhage and review of endoscopic management. Case Rep Gastrointest Med. 2014;2014:1-4.

7. Joarder Al, Faruque MS, Nur-E-Elahi M, Jahan I, Siddiqui O, Imdad S Islam MS, Ahmed HS, Haque MA. Dieulafoy's Lesion: An Overview.. Mymensingh Medical Journal: MMJ. 2014;23:186-94.

8. Khan R, Mahmad A, Gobrial M, Onwochei F. The diagnostic dilemma of Dieulafoy's lesion. Gastroenterology Res. 2015;8:201-6.

9. Enns R. Dieulafoy's lesions of the rectum: A rare cause of lower gastrointestinal bleeding. Can J Gastroenterol. 2001;15(8):541-5.

10. Souza JLS. Treatment of Dieulafoy's lesion of the right colon with epinephrine injection and argon plasma coagulation. Endoscopy. 2009; 41(Suppl. 2):E192.

11. Rivera Vaquerizo PA, Barajas Martínez JM, Blasco Colmenarejo M, Vicente Gutiérrez M, García García V, Pérez Flores R. Hemorragia digestiva baja por Dieulafoy de colon ascendente. Gastroenterol Hepatol. 2001;343-5.

12. Jain R, Chetty R. Dieulafoy disease of the colon. Arch Pathol Lab Med. 2009;133(11):1865-7.

13. Fukita Y. Treatment of a colonic Dieulafoy lesion with endoscopic hemoclipping. BMJ Case Rep. 2013 Apr 22;2013.

14. Sone Y, Nakano S, Takeda I, Kumada T, Kiriyama S, Hisanaga Y. Massive hemorrhage from a Dieulafoy lesion in the cecum: successful endoscopic management. Gastrointest Endosc [Internet]. 2000 Apr 1;51(4):5102. Available from: https://doi.org/10.1016/S0016-5107(00)70466-4.

15. Iacopini F, Petruzziello L, Marchese M, Larghi A, Spada C, Familiari P, et al. Hemostasis of Dieulafoy's lesions by argon plasma coagulation (with video). Gastrointest Endosc. 2007;66(1):20-6. 